\title{
An application of biregularity to quaternionic Lagrange interpolation
}

\author{
A. Perotti \\ Department of Mathematics, University of Trento, Via Sommarive, 14, I-38050 Povo Trento ITALY
}

\begin{abstract}
We revisit the concept of totally analytic variable of one quaternionic variable introduced by Delanghe [1] and its application to Lagrange interpolation by Güerlebeck and Sprössig [2]. We consider left-regular functions in the kernel of the Cauchy-Riemann operator

$$
\mathscr{D}=2\left(\frac{\partial}{\partial \bar{z}_{1}}+j \frac{\partial}{\partial \bar{z}_{2}}\right)=\frac{\partial}{\partial x_{0}}+i \frac{\partial}{\partial x_{1}}+j \frac{\partial}{\partial x_{2}}-k \frac{\partial}{\partial x_{3}} .
$$

For every imaginary unit $p \in \mathbf{S}^{2}$, let $\mathbf{C}_{p}=\langle 1, p\rangle \simeq \mathbf{C}$ and let $J_{p}=p_{1} J_{1}+p_{2} J_{2}+p_{3} J_{3}$ be the corresponding complex structure on $\mathbf{H}$. We identify totally regular variables with real-affine holomorphic functions from $\left(\mathbf{H}, J_{p}\right)$ to $\left(\mathbf{C}_{p}, L_{p}\right)$, where $L_{p}$ is the complex structure defined by left multiplication by $p$. We then show that every $J_{p}$-biholomorphic map, which is always a biregular function, gives rise to a Lagrange interpolation formula at any set of distinct points in $\mathbf{H}$.
\end{abstract}

Keywords: Quaternionic regular function, biregular function, Lagrange interpolation

PACS: 02.30.-f, 02.30.Fn, 02.60.Ed

\section{PRELIMINARIES}

We identify the space $\mathbf{C}^{2}$ with the set $\mathbf{H}$ of quaternions by means of the mapping that associates the pair $\left(z_{1}, z_{2}\right)=\left(x_{0}+\right.$ $\left.i x_{1}, x_{2}+i x_{3}\right)$ with the quaternion $q=z_{1}+z_{2} j=x_{0}+i x_{1}+j x_{2}+k x_{3} \in \mathbf{H}$. A quaternionic function $f=f_{1}+f_{2} j \in C^{1}(\Omega)$ is (left) regular (or hyperholomorphic) on $\Omega$ if

$$
\mathscr{D} f=2\left(\frac{\partial f}{\partial \bar{z}_{1}}+j \frac{\partial f}{\partial \bar{z}_{2}}\right)=\frac{\partial f}{\partial x_{0}}+i \frac{\partial f}{\partial x_{1}}+j \frac{\partial f}{\partial x_{2}}-k \frac{\partial f}{\partial x_{3}}=0 \quad \text { on } \Omega .
$$

We will denote by $\mathscr{R}(\Omega)$ the space of regular functions on $\Omega$ (cf. e.g. [8] and [7] for properties of these functions). The space $\mathscr{R}(\Omega)$ contains the identity mapping and every holomorphic mapping $\left(f_{1}, f_{2}\right)$ on $\Omega$ defines a regular function $f=f_{1}+f_{2} j$. The original definition of regularity given by Fueter (cf. [8] or [3]) differs from that adopted here by a real coordinate reflection. Let $\gamma$ be defined by $\gamma\left(z_{1}, z_{2}\right)=\left(z_{1}, \bar{z}_{2}\right)$. Then $f$ is regular on $\Omega$ if and only if $f \circ \gamma$ is Fueter-regular on $\gamma(\Omega)=\gamma^{-1}(\Omega)$.

A regular function $f \in C^{1}(\Omega)$ is called biregular if $f$ is invertible and $f^{-1}$ is regular.

\section{Holomorphic functions with respect to a complex structure $J_{p}$}

Let $J_{p}=p_{1} J_{1}+p_{2} J_{2}+p_{3} J_{3}$ be the orthogonal complex structure on $\mathbf{H}$ defined by a unit imaginary quaternion $p=p_{1} i+p_{2} j+p_{3} k$ in the sphere $\mathbf{S}^{2}=\left\{p \in \mathbf{H} \mid p^{2}=-1\right\}$. Let $\mathbf{C}_{p}=\langle 1, p\rangle$ be the complex plane spanned by 1 and $p$ and let $L_{p}$ be the complex structure defined on $T^{*} \mathbf{C}_{p} \simeq \mathbf{C}_{p}$ by left multiplication by $p$. If $f=f^{0}+i f^{1}: \Omega \rightarrow \mathbf{C}$ is a $J_{p}$-holomorphic function, i.e. $d f^{0}=J_{p}^{*}\left(d f^{1}\right)$ or, equivalently, $d f+i J_{p}^{*}(d f)=0$, then $f$ defines a regular function $\tilde{f}=f^{0}+p f^{1}$ on $\Omega$. We can identify $\tilde{f}$ with a holomorphic function

$$
\tilde{f}:\left(\Omega, J_{p}\right) \rightarrow\left(\mathbf{C}_{p}, L_{p}\right) .
$$

We have $L_{p}=J_{\gamma(p)}$, where $\gamma(p)=p_{1} i+p_{2} j-p_{3} k$. More generally, we can consider the space of holomorphic maps from $\left(\Omega, J_{p}\right)$ to $\left(\mathbf{H}, L_{p}\right)$

$$
\operatorname{Hol}_{p}(\Omega, \mathbf{H})=\left\{f: \Omega \rightarrow \mathbf{H} \text { of class } C^{1} \mid \bar{\partial}_{p} f=0 \text { on } \Omega\right\}=\operatorname{Ker}\left(\bar{\partial}_{p}\right)
$$


where $\bar{\partial}_{p}$ is the Cauchy-Riemann operator with respect to the structure $J_{p}$

$$
\bar{\partial}_{p}=\frac{1}{2}\left(d+p J_{p}^{*} \circ d\right) .
$$

For any positive orthonormal basis $\{1, p, q, p q\}$ of $\mathbf{H}\left(p, q \in \mathbf{S}^{2}\right)$, let $f=f_{1}+f_{2} q$ be the decomposition of $f$ with respect to the orthogonal sum

$$
\mathbf{H}=\mathbf{C}_{p} \oplus\left(\mathbf{C}_{p}\right) q .
$$

Let $f_{1}=f^{0}+p f^{1}, f_{2}=f^{2}+p f^{3}$, with $f^{0}, f^{1}, f^{2}, f^{3}$ the real components of $f$ w.r.t. the basis $\{1, p, q, p q\}$. Then the equations of regularity can be rewritten in complex form as

$$
\bar{\partial}_{p} f_{1}=J_{q}^{*}\left(\partial_{p} \bar{f}_{2}\right),
$$

where $\bar{f}_{2}=f^{2}-p f^{3}$ and $\partial_{p}=\frac{1}{2}\left(d-p J_{p}^{*} \circ d\right)$. Therefore every $f \in \operatorname{Hol}_{p}(\Omega, \mathbf{H})$ is a regular function on $\Omega$.

\section{The energy quadric}

In [4] and [6] was introduced the energy quadric of a regular function $f$. It is a family (depending on the point $z \in \Omega$ ) of positive semi-definite quadrics which contains a lot of information about the (Dirichlet) energy of $f$ and the holomorphicity properties of the function. In particular, this concept can be used to show that there are regular functions that are not $J_{p}$-holomorphic for any $p$, and that an affine biregular function is always $J_{p}$-biholomorphic for some $p$ : there exists $p$ such that $f \in \operatorname{Hol}_{p}(\mathbf{H}, \mathbf{H})$ and $f^{-1} \in \operatorname{Hol}_{\gamma(p)}(\mathbf{H}, \mathbf{H})$.

\section{TOTALLY REGULAR FUNCTIONS}

Definition 1 A regular function $f \in \mathscr{R}(\Omega)$ is called totally regular if the powers $f^{k}$ are regular on $\Omega$ for every integer $k \geq 0$ and $f^{k}$ is regular on $\Omega^{\prime}=\{x \in \Omega \mid f(x) \neq 0\}$ for every integer $k<0$.

Theorem 1 Let $f \in \mathscr{R}(\Omega)$ with image $\operatorname{Im}(f)$ contained in a (real) plane $H$. Then there exists $p \in \mathbf{S}^{2}$ such that $f \in \operatorname{Hol}_{p}(\Omega, \mathbf{H})$. If $f$ is non-constant, the complex structure $J_{p}$ is uniquely determined.

If $f=\sum_{\alpha=0}^{4} x_{\alpha} a_{\alpha}+b \in \mathscr{R}(\mathbf{H}), a_{\alpha}, b \in \mathbf{H}$, is (real) affine and $f$ has Jacobian matrix of maximum rank 2 , the same conclusion of Theorem 1 follows.

Corollary 2 If $f \in \mathscr{R}(\Omega)$ and $\operatorname{Im}(f)$ is contained in $\mathbf{C}_{p}$ for some $p \in \mathbf{S}^{2}$, then $f$ is a $\mathbf{J}_{p}$-holomorphic function, and therefore it is totally regular.

Remark 1 The decomposition $f=f_{1}+f_{2} q$ of a function $f \in \operatorname{Hol}_{p}(\Omega)$ w.r.t. any orthonormal basis $\{p, q, p q\}$ defines totally regular components $f_{1}, f_{2} \in \operatorname{Hol}_{p}\left(\Omega, \mathbf{C}_{p}\right)$.

We now prove the converse of Corollary 2 for affine functions. Using the energy quadric of a function, we are able to show that the regularity of $f$ and $f^{2}$ is sufficient to get that $f \in \operatorname{Hol}_{p}\left(\mathbf{H}, \mathbf{C}_{p}\right)$ and to obtain the total regularity of $f$.

Theorem 3 If $f \in \mathscr{R}(\mathbf{H})$ is affine and $f^{2}$ is regular, then $f$ has maximum rank 2 and there exists $p \in \mathbf{S}^{2}$ such that $f \in \operatorname{Hol}_{p}\left(\mathbf{H}, \mathbf{C}_{p}\right)$.

Corollary 4 If $f \in \mathscr{R}(\mathbf{H})$ is affine and $f^{2}$ is regular, then $f$ is totally regular.

The condition on the rank of $f$ given in Theorem 3 was proved, in the context of Fueter-regularity, in [2] $\$ 1.2$ (cf. also [3]§10). The preceding results tell that the set of affine totally regular functions coincides with the set

$$
\left\{f \text { affine } \mid f \in \cup_{p \in \mathbf{S}^{2}} \mathrm{Hol}_{p}\left(\mathbf{H}, \mathbf{C}_{p}\right)\right\} .
$$

Note that every subspace $\operatorname{Hol}_{p}\left(\mathbf{H}, \mathbf{C}_{p}\right)$ is a commutative algebra w.r.t. the pointwise product. 


\section{TOTALLY REGULAR VARIABLES AND BIREGULARITY}

Now our aim is to define, for any $p \in \mathbf{S}^{2}$, a totally regular function $v_{p} \in \operatorname{Hol}_{p}\left(\mathbf{H}, \mathbf{C}_{p}\right)$, which generalizes the concept of Fueter variables and totally analytic variables (cf. e.g. [3]§6.1).

Definition 2 Let $p \in \mathbf{S}^{2}$ and $\gamma(p)=p_{1} i+p_{2} j-p_{3} k$. Let $\vec{x}$ denote the vector part $\left(x_{1}, x_{2}, x_{3}\right)$ of $x \in \mathbf{H}$. We set

$$
v_{p}(x)=x_{0}+(\overrightarrow{\gamma(p)} \cdot \vec{x}) p
$$

In particular, we get the variables $v_{i}=x_{0}+x_{1} i=z_{1} \in H_{i o l}\left(\mathbf{H}, \mathbf{C}_{i}\right), v_{j}=x_{0}+x_{2} j \in H_{o l}\left(\mathbf{H}, \mathbf{C}_{j}\right), v_{k}=x_{0}-x_{3} k \in$ $\operatorname{Hol}_{k}\left(\mathbf{H}, \mathbf{C}_{k}\right)$. We can also consider the totally regular variables $v_{p}^{\prime}=v_{p} p \in \operatorname{Hol}_{p}\left(\mathbf{H}, \mathbf{C}_{p}\right)$, which satisfy the additive property

$$
\frac{1}{|p+q|}\left(v_{p}^{\prime}+v_{q}^{\prime}\right)=v_{\frac{p+q}{|p+q|}}^{\prime} \in \operatorname{Hol}_{p+q}\left(\mathbf{H}, \mathbf{C}_{p+q}\right)
$$

For every $a \in \mathbf{H}, a \neq 0$, let $\operatorname{rot}_{a}(q)=a q a^{-1}$ be the three-dimensional rotation of $\mathbf{H}$ defined by $a$. In [5] was studied the effect of rotations on regularity and holomorphicity of functions. As an application of those results, we get that $v_{p}$ can be seen as one component of a biregular function.

Theorem 5 a) For every $p \in \mathbf{S}^{2}$, the function $v_{p}$ is regular on $\mathbf{H}$ and belongs to the space $\operatorname{Hol}_{p}\left(\mathbf{H}, \mathbf{C}_{p}\right)$. Therefore $v_{p}$ is totally regular.

b) For any $p, q \in \mathbf{S}^{2}, q \perp p$, let $a \in \mathbf{H}$ be such that $\operatorname{rot}_{\gamma(a)}(i)=p, \operatorname{rot}_{\gamma(a)}(j)=q$. There exists an affine biregular function $f_{a}=v_{p}+w_{a} q$, with totally regular components $v_{p}, w_{a} \in \operatorname{Hol}_{p}\left(\mathbf{H}, \mathbf{C}_{p}\right)$. The function $f_{a} \in \operatorname{Hol}_{p}(\mathbf{H}, \mathbf{H})$ is $J_{p}$-biholomorphic, with inverse of the same type as $f_{a}$ :

$$
f_{a}^{-1}=f_{a^{\prime}}=v_{\gamma(p)}+w_{a^{\prime}} q^{\prime} \in \operatorname{Hol}_{\gamma(p)}(\mathbf{H}, \mathbf{H}) \quad\left(a^{\prime}=\gamma(a)^{-1}, \gamma(p)=\operatorname{rot}_{a}^{-1}(i), q^{\prime}=\operatorname{rot}_{a}^{-1}(j)\right)
$$

Remark 2 The biregular function $f_{a}$ is defined by the simple formula $f_{a}=\operatorname{rot}_{\gamma(a) a}$.

\section{QUATERNIONIC LAGRANGE INTERPOLATION}

In [2], as an application of totally analytic variables, a Lagrange's Interpolation Theorem was proved. Given $k$ distinct points $b_{1}, \ldots, b_{k} \in \mathbf{H}$ and $k$ values $u_{1}, \ldots, u_{k} \in \mathbf{H}$, one wants to construct a Lagrange polynomial in the module of regular functions, i.e. a polynomial $L \in \mathscr{R}(\mathbf{H})$ such that $L\left(b_{j}\right)=u_{j}$ for every $j=1, \ldots, k$.

Theorem 6 Given a $J_{p}$-biholomorphic mapping $f=f_{1}+f_{2} q \in \operatorname{Hol}_{p}(\mathbf{H}, \mathbf{H})(q \perp p)$, there exist (infinitely many) $\alpha, \beta \in \mathbf{C}_{p}$ such that $g=\alpha f_{1}+\beta f_{2} \in \operatorname{Hol}_{p}\left(\mathbf{H}, \mathbf{C}_{p}\right)$ is totally regular and satisfies the conditions

$$
g\left(b_{i}\right) \neq g\left(b_{j}\right) \quad \forall i \neq j(i, j=1, \ldots, k) .
$$

The numbers $\alpha, \beta$ can also be found in the real field.

Then every $J_{p}$-biholomorphic mapping $f$ gives rise to a Lagrange interpolation function (a polynomial if $f$ is a polynomial function), given by the formula

$$
L=\sum_{s=1}^{k} l_{s} u_{s}, \quad \text { where } \quad l_{s}(x)=\prod_{t \neq s}\left(g(x)-g\left(b_{t}\right)\right)\left(g\left(b_{s}\right)-g\left(b_{t}\right)\right)^{-1} \in \operatorname{Hol}_{p}\left(\mathbf{H}, \mathbf{C}_{p}\right) .
$$

The functions $l_{s}^{m}$ are regular on $\mathbf{H}$ for every integer $m>0$ and $L \in \mathscr{R}(\mathbf{H})$. The powers of $L$ are regular if also the values $u_{s}$ belong to the subalgebra $\mathbf{C}_{p}$.

Example 1 If we take the function $f_{a}$ of Theorem 5 as $J_{p}$-biholomorphic mapping, and $\alpha, \beta \in \mathbf{R}$, then $g=\alpha f_{1}+\beta f_{2}$ is the linear function

$$
\operatorname{rot}_{\gamma(a)} \circ\left(\alpha z_{1}+\beta z_{2}\right) \circ \operatorname{rot}_{a} \text {. }
$$




\section{ACKNOWLEDGMENTS}

The work was partially supported by MIUR (PRIN Project "Proprietà geometriche delle varietà reali e complesse") and GNSAGA of INdAM.

\section{REFERENCES}

1. R. Delanghe, Math. Ann., 185, 91-111 (1970).

2. K. Gürlebeck and W. Sprössig, Quaternionic analysis and elliptic boundary value Problems, Birkhäuser, Basel, 1990.

3. K. Gürlebeck, K. Habetha and W. Sprössig, Holomorphic functions in the plane and n-dimensional space. Translated from the 2006 German original Funktionentheorie in Ebene und Raum, Birkhäuser Verlag, Basel, 2008.

4. A. Perotti, "Holomorphic functions and regular quaternionic functions on the hyperkähler space $\mathbf{H}$ ", in Proceedings of the 5th ISAAC Congress, Catania 2005, World Scientific Publishing Co. (in press) (arXiv:0711.4440v1).

5. A. Perotti, "Directional quaternionic Hilbert operators," in Hypercomplex analysis, edited by I. Sabadini, M. Shapiro and F. Sommen, Birkhäuser, Basel, in press.

6. A. Perotti, Every biregular function is biholomorphic, Advances in Applied Clifford Algebras, in press.

7. M.V. Shapiro and N.L. Vasilevski, Complex Variables Theory Appl.. 27 no.1, 17-46 (1995).

8. A. Sudbery, Mat. Proc. Camb. Phil. Soc., 85, 199-225 (1979). 